\title{
Towards actively stabilized micro ring resonator based frequency combs
}

\author{
Henriksen, M. R.; Kamel, Ayman Nassar; Pu, Minhao; Yvind, Kresten; Thomsen, Jan Werner
}

Published in:

2017 Conference on Lasers and Electro-Optics (CLEO)

Link to article, DOI:

10.1364/CLEO_QELS.2017.JTh2A.88

Publication date:

2017

Document Version

Peer reviewed version

Link back to DTU Orbit

Citation (APA):

Henriksen, M. R., Kamel, A. N., Pu, M., Yvind, K., \& Thomsen, J. W. (2017). Towards actively stabilized micro ring resonator based frequency combs. In 2017 Conference on Lasers and Electro-Optics (CLEO) [JTh2A.88] IEEE. https://doi.org/10.1364/CLEO_QELS.2017.JTh2A.88

\section{General rights}

Copyright and moral rights for the publications made accessible in the public portal are retained by the authors and/or other copyright owners and it is a condition of accessing publications that users recognise and abide by the legal requirements associated with these rights.

- Users may download and print one copy of any publication from the public portal for the purpose of private study or research.

- You may not further distribute the material or use it for any profit-making activity or commercial gain

- You may freely distribute the URL identifying the publication in the public portal 


\title{
Towards Actively Stabilized Micro Ring Resonator Based Frequency Combs
}

\author{
M. R. Henriksen ${ }^{1}$, A. N. Kamel ${ }^{2}$, M. Pu ${ }^{2}$, K. Yvind ${ }^{2}$, J. W. Thomsen ${ }^{1}$ \\ ${ }^{1}$ Niels Bohr Institute, Blegdamsvej 17, DK-2100 Copenhagen, Denmark \\ ${ }^{2}$ DTU Fotonik, Technical University of Denmark, Building 343, DK-2800 Kgs. Lyngby, Denmark \\ romme@nbi.ku.dk
}

\begin{abstract}
We present a simple and versatile scheme for active locking of a micro ring resonance to a highly stable fibre laser allowing continuous resonance tuning and locking on a time scale of 10 microseconds.
\end{abstract}

OCIS codes: $190.4390,230.5750$.

The quest for high stability and accuracy of modern chip based frequency combs is key for demanding applications in metrology [1,2]. Great progress has been demonstrated for chip-based Kerr frequency combs, yet challenges remain to be addressed in terms of phase stability and accuracy. With the recent introduction of soliton combs significant advances have been made in terms of low phase noise and overall comb coherence. For optimal operation of the soliton comb the micro ring resonator's (MRR) resonance must be locked to the pump laser frequency at some detuning, possibly changing the detuning on a typical time scale of a few milliseconds. Usually, this is carried out by a trial and error procedure: tuning the scan speed of the pump laser with respect to the thermally induced drift of the MRR [3].

Here we present an AlGaAs-on-insulator MRR [4] with an integrated heater enabling fast tuning of the MRR resonance. The MRR is pumped with a fixed frequency low phase noise CW fibre laser and locked to the pump laser frequency using the Pound-Drever-Hall technique. This gives a high degree of control on the detuning between the pump laser frequency and MRR resonance on a time scale of microseconds.

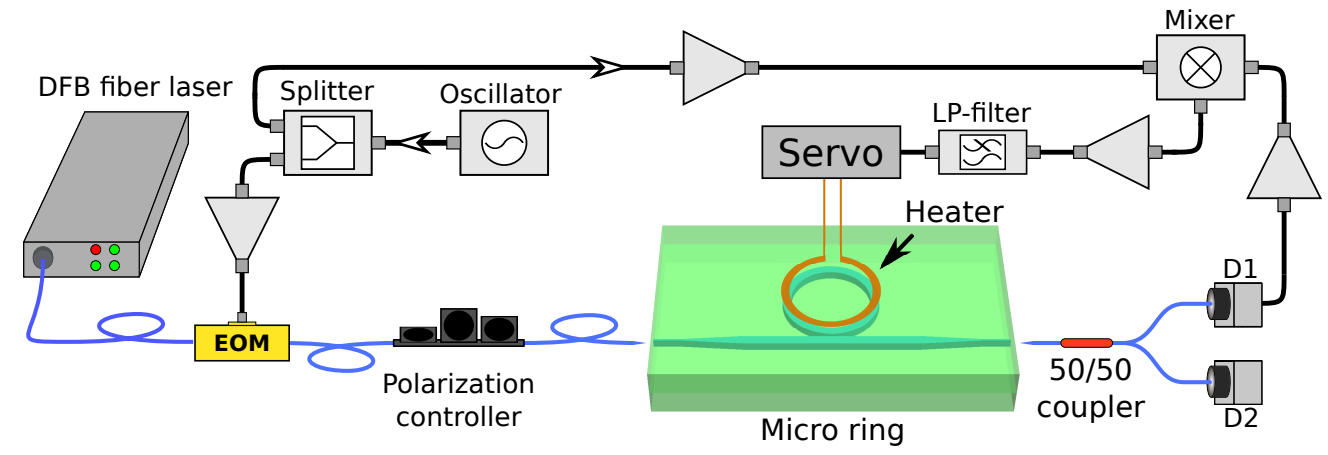

Fig. 1: Schematic of the set-up. The DFB fibre laser output is phase modulated at $5.26 \mathrm{GHz}$ by an electro-optic modulator (EOM). The waveguide transmission is coupled to a fast (D1) and a slow (D2) photo detector. The signal from D1 is demodulated in the mixer and used as an error signal to control the heater current and thereby the MRR resonance frequency. Detector D2 is used to monitor the MRR transmission shown in fig. 2a.

The set-up is illustrated in fig. 1. The optical output power of the pump laser is attenuated to $0.5 \mathrm{~mW}$ in order to minimize optically induced thermal distortion of the line shape measurement. Before coupling into the chip waveguide, an electro-optic modulator (EOM) is used for phase modulation. The chip transmission is coupled through a 50/50 fibre splitter and monitored on a fast and a slow photo detector. A measurement of the MRR line shape shows a resonance linewidth of $3.8 \mathrm{GHz}$ corresponding to a intrinsic Q-value of 50.000. The EOM generates sidebands at $5.26 \mathrm{GHz}$ which is used for phase sensitive detection of the detuning between the pump laser and the resonator. This is possible as only the central line couples to the resonator and experiences the resulting phase shift. The beat-note between the central line and the sidebands is detected with the fast detector (D1) and is demodulated using a mixer to generate a 
signal that is odd with respect to the frequency detuning (fig. 2b). This signal is filtered through a $7.3 \mathrm{kHz}$ low-pass filter and used as an error input signal on a P-I-I servo system controlling the heater current.

The system's stability is determined by an in-loop measurement of the error signal (fig. 2c). The measurement shows an achieved long term stability of $600 \mathrm{kHz}$. The measured stability corresponds to a line-splitting of approximately 6500. The stability measurement is limited by the signal-to-noise ratio of the error signal due to the low pump power.

For more realistic MRR Q-values, a factor of ten greater than presented here, the achievable stability will be a factor of 10 better than presented here. Such a system will facilitate the generation of a soliton comb on a more robust and repeatable basis. This technique, is to our knowledge, the first that offers the possibility of simultaneous pumping and locking of a MRR with an absolute optical frequency reference, moving MRR based frequency combs into a new stability regime.

The authors acknowledge financial support from the Danish Research Council and Villum Fonden via the SPOC (DNRF123), and NATEC centers.

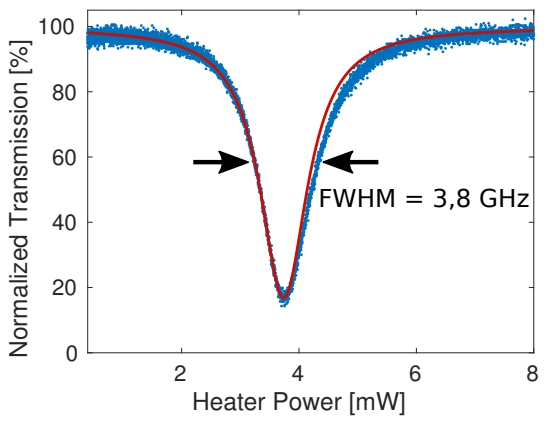

(a)

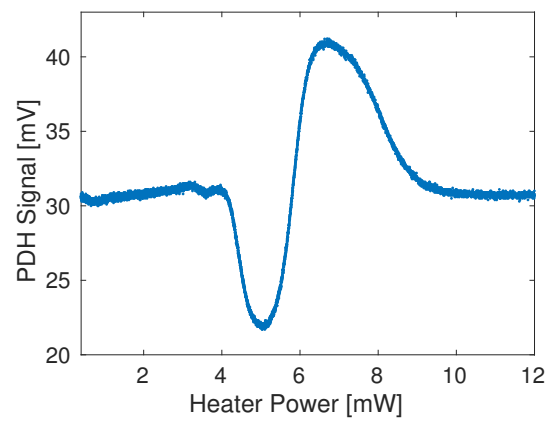

(b)

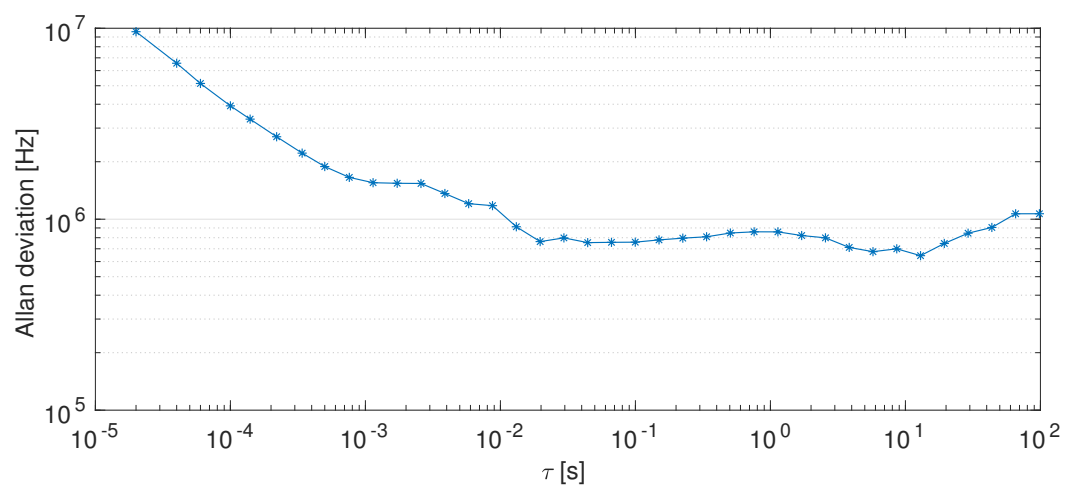

(c)

Fig. 2: (a) Transmission signal. A Lorentzian line shape is fitted and the FWHM of $3.8 \mathrm{GHz}$ is indicated. (b) PoundDrever-Hall signal used as error signal for servo system. (c) Allan deviation of an in-loop measurement on the error signal showing a long term stability of $600 \mathrm{kHz}$.

\section{References}

1. Del'Haye, P., et al. "Phase-coherent microwave-to-optical link with a self-referenced microcomb." Nature Photonics (2016).

2. Huang, S.-W., et al. "Phase stabilization of Kerr frequency comb internally without nonlinear optical interferometry." arXiv preprint arXiv:1611.02858 (2016).

3. Herr, T., et al. "Temporal solitons in optical microresonators.", Nature Photonics 8.2 (2014): 145-152.

4. Ottaviano, L., et al. "Low-loss high-confinement waveguides and microring resonators in AlGaAs-on-insulator." Optics Letters 41.17 (2016): 3996-3999. 